\title{
STABILITY OF PULSES IN NONLINEAR OPTICAL FIBERS USING PHASE-SENSITIVE AMPLIFIERS
}

\author{
J. NATHAN KUTZ ${ }^{\dagger}$ AND WILLIAM L. KATH ${ }^{\ddagger}$
}

\begin{abstract}
This paper considers the stability of soliton-like pulses propagating in nonlinear optical fibers with periodically-spaced phase-sensitive amplifiers, a situation where the averaged pulse evolution is governed by a fourth-order nonlinear diffusion equation similar to the KuramotoSivashinsky or Swift-Hohenberg equations. A bifurcation and stability analysis of this averaged equation is carried out, and in the limit of small amplifier spacing, a steady-state pulse solution is shown to be asympotically stable. Furthermore, both a saddle-node bifurcation and a subcritical bifurcation from the zero solution are found. Analytical results are confirmed using the bifurcation software package AUTO. The analysis provides evidence for the existence of stable pulse solutions for a wide range of parameter values, including those corresponding to physically realizable soliton communications systems.
\end{abstract}

Key words. solitons, nonlinear optical pulse propagation, optical fibers, bifurcation theory

AMS subject classifications. 35Q55,35B32,35B20,78 A60,41A60

1. Introduction. Pulse propagation in nonlinear optical fibers has attracted much interest in recent years due to the potentially large increase in transmission speed which is likely to be obtained by the use of optical solitons. Solitons are ideal candidates for optical 'bit' carriers due to their robust nature and non-dispersive behavior $[1,2,3]$. When communication distances longer than a few tens of kilometers are considered, however, a propagating pulse must be periodically amplified in order to compensate for the intrinsic residual attenuation present in the fiber. For typical pulse widths, the evolution lengthscale (measured by either the dispersion length or the soliton period) is much longer than the required amplifier spacing. The loss and amplification are therefore strong, periodic and rapidly-varying perturbations to the governing evolution equation. In this case, the goal is to average over the fluctuations induced by these rapid perturbations and determine the effective or averaged evolution of the propagating pulse over long distances.

Periodically-spaced erbium-doped fiber amplifiers $[4,5,6,7,8]$ have been shown to be one effective method for compensating loss in such communication systems. These amplifiers are relatively short sections (e.g., 1 to 2 meter) of optical fiber which are doped with small amounts of the rare-earth element erbium and are pumped with a diode laser. The optical pumping induces a population inversion in the erbium atoms which provides signal gain via the stimulated emission of light. The population inversion also necessarily contributes spontaneous emission noise to the signal, however, and this noise (along with other noise sources [9, 10]) decreases the maximum allowable bit rate by introducing perturbations to the various soliton parameters. Most importantly, perturbations to the soliton group-velocity (or equivalently, the soliton central frequency) generate a random walk in the soliton position known as Gordon-Haus timing jitter [11, 12]. This timing jitter can be significantly reduced by the addition of frequency filters $[13,14,15]$ to the optical fiber line. Such filters add a frequency reference and a restoring force in the frequency domain which offset the

\footnotetext{
$\dagger$ Institute of Mathematics and its Applications, University of Minnesota, 206 Church St., Minneapolis, MN 55455

$\ddagger$ Engineering Sciences and Applied Mathematics, McCormick School of Engineering and Applied Science, Northwestern University, 2145 Sheridan Rd., Evanston, IL 60208-3125
} 
random perturbations. Direct timing jitter is still present, however, due to random perturbations of the soliton position [12].

As an alternative to erbium-doped amplifiers, the use of phase-sensitive amplifiers (PSAs) for compensating the linear loss in the fiber has been proposed [16]. PSAs have been suggested since they are free of spontaneous emission noise and are ideal quantum limited amplifiers with a 0dB noise figure [16]. PSAs thus do not contribute to the Gordon-Haus timing jitter, and as a consequence, a significant increase in the maximum allowable bit-rate has been predicted [17, 18]. Generally speaking, PSAs amplify light with light. In particular, phase-sensitive gain can be provided to an optical signal by an optical pump pulse through the process of parametric amplification [19]. As a result, the components of the signal pulse which are in-phase with the pump pulse are amplified, while out-of-phase components are attenuated. This attenuation of the out-of-phase portion works to eliminate phase variations across the signal pulse's profile, which in turn leads to an enhanced stability of the signal pulse $[20,21]$.

The aim of this paper is to present a detailed analysis of the fourth-order, nonlinear diffusion equation which has been derived to describe the averaged pulse dynamics in a nonlinear optical fiber where linear loss in the fiber is compensated by a chain of periodically spaced, phase-sensitive amplifiers [20, 21]. This averaged evolution equation, which is similar to the Kuramoto-Sivashinsky and Swift-Hohenberg equations [22], supports stable pulse solutions with steady-state solutions acting as attractors for a wide range of initial conditions.

The paper is arranged in the following manner. Section 2 gives a brief summary of the derivation of the averaged fourth-order equation. In Section 3 , the linear stability of a propagating pulse is investigated in the limit of small amplifier spacing. Section 4 presents a bifurcation analysis for the trivial solution of the averaged equation which is closely tied with results in Section 5. Section 5 explores steady-state solutions in relevant ranges of parameter space through use of the bifurcation software AUTO. The paper is concluded in Section 6 which summarizes the major results of the analysis.

2. Governing Equations. Pulse propagation through an optical fiber which includes dispersion, nonlinearity, linear loss, and periodic phase-sensitive amplification is governed by the perturbed nonlinear Schrödinger (NLS) equation [2, 20, 21]

$$
q_{Z}=\frac{i}{2} q_{T T}+i|q|^{2} q-\Gamma q+\frac{1}{\epsilon} h\left(\frac{Z}{\epsilon}\right) q+\frac{1}{\epsilon} e^{i \phi(Z)} f\left(\frac{Z}{\epsilon}\right) q^{*}
$$

where $\Gamma$ is the linear loss rate in the fiber and the rapidly varying periodic functions $h$ and $f$ account for the effect of the optical phase-sensitive gain of the lumped amplifiers $[20,21]$. Here the phase of the amplifiers is represented by $\phi(Z)$. The length $Z$ in $(1)$ has been scaled on a typical dispersion length $Z_{0}$ (e.g., $200-500 \mathrm{~km}$ ); the amplifier spacing $Z_{l}$ is assumed to be much shorter than this length (e.g., $Z_{l}=20-50 \mathrm{~km}$ ). Mathematically, this assumption is made by defining $\epsilon l \equiv Z_{l} / Z_{0}$ and taking $\epsilon \ll 1$ with $l \sim O(1)[20,21]$.

Performing a multiple scale averaging [23, 24] of (1) using the short length scale $\zeta=Z / \epsilon$, the dispersion length scale $Z$, and the long length scale $\xi=\epsilon Z$ gives the following fourth-order evolution equation [20, 21]

$$
U_{\bar{\xi}}+\frac{1}{4}\left(\partial_{T}^{2}-\kappa\right)^{2} U-\Delta \alpha U-\kappa U^{3}+U^{5}+3 B U U_{T}^{2}+(B+1) U^{2} U_{T T}=0,
$$


where $B=(2-\tanh \Gamma l / \Gamma l), \kappa=d \phi / d Z, \bar{\xi}=\left(\frac{l}{2} \operatorname{coth} \Gamma l\right) \xi$ and $U$ is the scaled in-phase component of the pulse envelope after each amplifier, namely

$$
U=\left(\frac{1-e^{-2 \Gamma l}}{2 \Gamma l}\right)^{1 / 2} \Re e\left(q e^{-i \phi / 2}\right)
$$

Equation (2) is a fourth-order, nonlinear, diffusion equation which governs the pulse dynamics over the long length scale $\bar{\xi}$. The parameter $\Delta \alpha$ represents an $O\left(\epsilon^{2}\right)$ deviation from the exact balance of loss and gain which accounts for a small necessary overamplification $(\Delta \alpha>0)$. The parameter $\kappa$, which represents a constant amplifier phase rotation rate, can be taken to be unity without loss of generality since it can be scaled out of (2). Therefore, $\kappa=1$ for the remainder of this paper.

Note that the amplitude rescaling given in (3) is the same rescaling used when considering soliton propagation with erbium-doped amplifiers, i.e., the guiding-center soliton rescaling $[5,6,7]$. This amplitude rescaling corresponds to normalizing the pulse envelope so that $\int U^{2} d T$ is the average pulse energy over one amplification period [20,21]. Although the evolution equation (2) inherits much of its structure from the NLS equation, it is important to note that the evolution is of a non-Hamiltonian nature, and therefore, its dynamics are those of a dissipative system.

3. Pulse solutions and their stability. In general, a full understanding of the stability and dynamics of pulse solutions of (2) can only be obtained numerically. The aim here, however, is to investigate analytically the parameter regimes of the averaged evolution accessible via asymptotic and perturbation methods. It will be shown in Section 5 that the qualitative features of the results remain valid outside of the asymptotic regimes explored.

An idea of the general structure of the solutions to (2) can be obtained by considering $\Gamma l=0$. In this limit, $U=\eta \operatorname{sech} \eta T$ where $\eta=\left(1 \pm(\Delta \alpha)^{1 / 2}\right)^{1 / 2}$. These solutions are plotted in Figure 1. In this section, we investigate the effect of the perturbation resulting when $\Gamma l$ is nonzero upon solutions near the fold or limit point in the bifurcation diagram (region A in Figure 1), and also determine their stability. The limit of small $\Gamma l$ and $\Delta \alpha$ corresponds physically to assuming that both the amplifier spacing and the amount of overamplification are small.

Since the parameter $B$ in (2) is an even function of $\Gamma l$, we let $\delta=(\Gamma l)^{2} \ll 1$ and expand

$$
\begin{aligned}
U & =U_{0}+\delta U_{1}+\delta^{2} U_{2}+\ldots \\
\Delta \alpha & =\delta \alpha_{1}+\delta^{2} \alpha_{2}+\ldots
\end{aligned}
$$

In addition, we define the length scale $\sigma=\delta \bar{\xi}$ and use a multiple-scale expansion to capture any slow growth in $U$ caused by the perturbation.

At leading order in $\delta, O(1),(2)$ gives

$$
U_{0 \bar{\xi}}+\left(-\frac{1}{2} \partial_{T}^{2}-U_{0}^{2}+\frac{1}{2}\right)^{2} U_{0}=0 .
$$

It is easy to show that the hyperbolic secant solution $U_{0}=\operatorname{sech} T$ is a steady-state solution of (5). The shape of this pulse agrees with what is expected physically as $\delta$ approaches zero - a limit where the fluctuations caused by the attenuation and phase-sensitive amplification are negligible - namely, the hyperbolic secant shape 


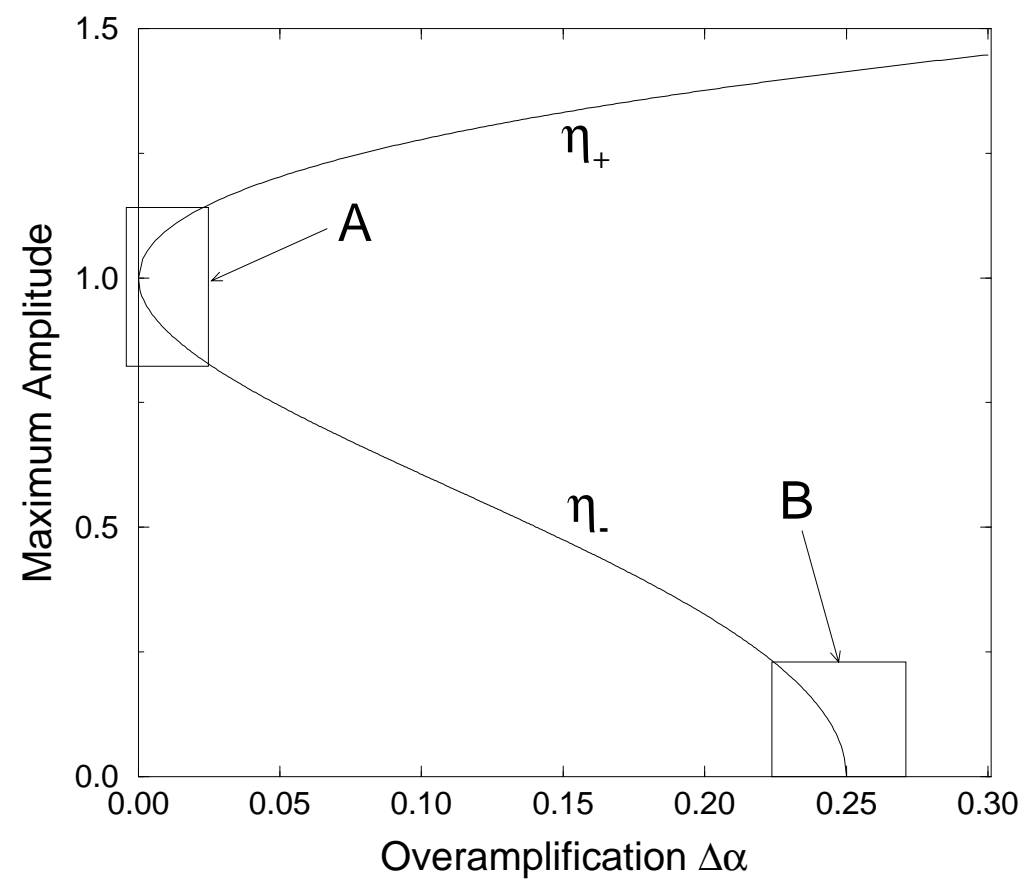

FIG. 1. Plot of the maximum amplitude for the two solution branches $U=\eta \operatorname{sech} \eta T$ where $\eta=\left(1 \pm 2(\Delta \alpha)^{1 / 2}\right)^{1 / 2}$. Region $A$ is the asymptotic regime explored in Section 3 and region $B$ is the asymptotic regime explored in Section 4.

associated with a soliton solution of the nonlinear Schrödinger equation. The form of (5) is clearly inherited from the underlying NLS equation.

Writing (2) as

$$
U_{\bar{\xi}}+N(U)=0
$$

when expanded in powers of $\delta$ using (4) it is found that

$$
\begin{aligned}
N(U)= & \left(-\frac{1}{2} \partial_{T}^{2}-U_{0}^{2}+\frac{1}{2}\right)^{2} U_{0}+\delta\left[L_{-} L_{+} U_{1}-H_{1}\left(U_{0}, \alpha_{1}\right)\right] \\
& +\delta^{2}\left[L_{-} L_{+} U_{2}-H_{2}\left(U_{0}, U_{1}, \alpha_{1}, \alpha_{2}\right)\right]+\cdots
\end{aligned}
$$

where $H_{n}$ represents the perturbing terms at $O\left(\delta^{n}\right)$ which appear in the equation for $U_{n}$, and the operators $L_{-}$and $L_{+}$are the real and imaginary parts of the linearized NLS equation [25],

$$
L_{+}=-\frac{1}{2} \partial_{T}^{2}-3 U_{0}^{2}+\frac{1}{2}, \quad L_{-}=-\frac{1}{2} \partial_{T}^{2}-U_{0}^{2}+\frac{1}{2} .
$$

The self-adjoint operators $L_{+}$and $L_{-}$are well understood [25]. The spectrum of $L_{-}$contains a single discrete eigenvalue at $\lambda=0$, while the spectrum of $L_{+}$contains two discrete eigenvalues, one at $\lambda=-3$ and the other at $\lambda=0$. Both operators also have a continuous spectrum starting at $\lambda=1$. The associated eigenfunctions of the operators are found by noting

$$
L_{-}(\operatorname{sech} T)=0
$$




$$
\begin{aligned}
L_{+}(\operatorname{sech} T \tanh T) & =0, \\
L_{+}\left(\operatorname{sech}^{2} T\right) & =-3 \operatorname{sech}^{2} T .
\end{aligned}
$$

Since $L_{-}$and $L_{+}$do not commute, the linearized operator $-L_{-} L_{+}$is nonselfadjoint and less straightforward to analyze. Using the properties of $L_{-}$and $L_{+}$ [25], however, it can be shown that $-L_{-} L_{+}$contains two zero eigenvalues with eigenfunctions given by

$$
\begin{aligned}
L_{-} L_{+}(\operatorname{sech} T \tanh T) & =0, \\
L_{-} L_{+}(\operatorname{sech} T-T \tanh T \operatorname{sech} T) & =0 .
\end{aligned}
$$

The corresponding zero eigenfunctions of the adjoint operator $-L_{+} L_{-}$, which are important in determining the appropriate solvability conditions associated with the forcings $H_{n}$, are given by

$$
\begin{aligned}
L_{+} L_{-}(\operatorname{sech} T) & =0, \\
L_{+} L_{-}(T \operatorname{sech} T) & =0 .
\end{aligned}
$$

Returning now to the perturbation expansion at $O(\delta)$, it is found that

$$
U_{1 \bar{\xi}}+L_{-} L_{+} U_{1}=H_{1}\left(U_{0}, \alpha_{1}\right)=-U_{0} U_{0 T}^{2}-\frac{1}{3} U_{0}^{2} U_{0 T T}+\alpha_{1} U_{0} .
$$

We will show shortly that, except for initial conditions which lie in the space spanned by the two zero eigenfunctions, all solutions of the homogeneous part of the linearized equation (7) decay exponentially. To determine the stability of (2), therefore, it is only necessary to determine the effect of the perturbation upon the two zero eigenvalues. One of these eigenvalues arises due to the translation invariance of (5), i.e., $\operatorname{sech} T \tanh T$ when $\Gamma l=0$, but since the full equation (2) is also invariant under translations, this zero eigenvalue must necessarily persist under the perturbation and thus is not relevant to the present stability analysis. The other zero eigenvalue (the one associated with the eigenfunction sech $T-T \tanh T \operatorname{sech} T$ ) is affected by the perturbation, however, and therefore the stability of the pulse is determined solely by this eigenvalue. Note that at $\Gamma l=0$ the zero mode arises from from an infinitesimal amplitude invariance due to the vertical tangent at the saddle-node bifurcation (Region A of Fig. 1).

Since (7) has homogeneous solutions, solvability conditions are required in order for a solution of the perturbed problem to exist [26], namely it is necessary that the forcing $H_{1}$ be orthogonal to the null space of the adjoint linearized operator. A condition is therefore associated with each of the zero adjoint eigenmodes. These are

$$
\begin{aligned}
\left(H_{1}, \operatorname{sech} T\right) & =0, \\
\left(H_{1}, T \operatorname{sech} T\right) & =0,
\end{aligned}
$$

where $(h, g)=\int_{-\infty}^{\infty} h g d T$ denotes the inner product. Using the explicit form of $H_{1}$, it is found that the second condition, $(8 \mathrm{~b})$, is automatically satisfied, but the first condition, (8a), is only satisfied if $\alpha_{1}=0$. With $\alpha_{1}=0$, the solution for $U_{1}$ which is even and independent of $\bar{\xi}$ is then

$$
U_{1}=-(1 / 6) \operatorname{sech} T+\beta(\sigma)(\operatorname{sech} T-T \tanh T \operatorname{sech} T),
$$


where $\beta(\sigma)$ is arbitrary at this order.

At second order, $O\left(\delta^{2}\right)$, the situation is similar, except that $H_{1}$ in (8) must be replaced by

$$
\begin{aligned}
H_{2}= & -\alpha_{2} U_{0}-U_{1 \sigma}+3 U_{0} U_{1}^{2}-10 U_{0}^{3} U_{1}^{2}-3 U_{0} U_{1 T}^{2}-6 U_{0 T} U_{1 T} U_{1} \\
& -2 U_{1}^{2} U_{0 T T}-4 U_{0} U_{1} U_{1 T T}-2 U_{0} U_{0 T} U_{1 T}-U_{0 T}^{2} U_{1}-\frac{1}{3} U_{0}^{2} U_{1 T T} \\
& -\frac{2}{3} U_{0} U_{1} U_{0 T T}+\frac{2}{5} U_{0} U_{0 T}^{2}+\frac{2}{15} U_{0}^{2} U_{0 T T} .
\end{aligned}
$$

At this order the solvability condition (8a) gives an equation for $\beta(\sigma)$,

$$
\frac{d \beta}{d \sigma}=2 \alpha_{2}-2 \beta^{2}+\frac{8}{9} \beta-\frac{16}{135} .
$$

This equation has both a stable and an unstable steady-state solution for $\alpha_{2}>$ (4/405). This means that when $\Gamma l$ is small and, equivalently, $\Delta \alpha>(4 / 405)(\Gamma l)^{4} \equiv$ $\Delta \alpha_{c}$, a stable steady-state pulse solution of (2) exists.

The critical value $\Delta \alpha_{c}$ determines the minimum amount of overamplification necessary for stable pulse solutions to exist. The need for a small amount of overamplification is consistent with the use of PSAs since there is a small amount of energy lost due to the attenuation of the out-of-phase quadrature. For values of $\Delta \alpha$ below $\Delta \alpha_{c}$, pulses decay to zero. Of course, these results are only valid when $\Gamma l$ is small, but they are nonetheless indicative of the results obtained using numerical simulations for values of $\Gamma l$ which are $O(1)[20,21]$.

It remains to be verified that considering perturbations of the two zero eigenvalues of $-L_{-} L_{+}$is sufficient to determine the stability of pulse solutions of (2). This will be done by showing that solutions of the homogeneous equation

$$
U_{1 \bar{\xi}}+L_{-} L_{+} U_{1}=0
$$

decay exponentially for all initial conditions which do not lie in the space spanned by the two zero eigenfunctions. The exponential decay rate will by shown to be $O(1)$, so that for sufficiently small perturbations this decay rate will still be bounded away from zero. Restricting the initial condition for $(10)$ is accomplished by requiring it to be orthogonal to the two zero adjoint eigenfunctions,

$$
\begin{aligned}
\left(U_{1}, \operatorname{sech} T\right) & =0, \\
\left(U_{1}, T \operatorname{sech} T\right) & =0 .
\end{aligned}
$$

It is straightforward to verify that if these conditions are satisfied at $\bar{\xi}=0$ then they hold for all $\bar{\xi}$.

The exponential decay of solutions constrained by (11) is shown by considering

$$
V(\bar{\xi})=\left(U_{1}, L_{+} U_{1}\right)
$$

Weinstein [25] shows that, subject to the constraints given in (11),

$$
\left(U_{1}, L_{+} U_{1}\right) \geq C_{1}\left\|U_{1}\right\|^{2},
$$

where $\left\|U_{1}\right\|^{2}=\left(U_{1}, U_{1}\right)$ is the $L^{2}$ norm and $C_{1}$ is a positive constant. Thus, $V$ is positive definite. In addition, using (12) and (10), as well as the self-adjointness of $L_{-}$and $L_{+}$,

$$
\frac{d V}{d \bar{\xi}}=-2\left(U_{1}, L_{+} L_{-} L_{+} U_{1}\right)
$$


The right-hand-side of (14) can also be written

$$
\left(U_{1}, L_{+} L_{-} L_{+} U_{1}\right)=\left(W, L_{-} W\right)
$$

where $W=L_{+} U_{1}$.

Since $W=L_{+} U_{1}$, this means that $(W, \operatorname{sech} T \tanh T)=0$. We can also then rewrite the constraint (11a) in terms of $W$ as

$$
\left(U_{1}, \operatorname{sech} T\right)=\left(L_{+}^{-1} W, \operatorname{sech} T\right)=\left(W, L_{+}^{-1} \operatorname{sech} T\right)=0 .
$$

While the inverse of $L_{+}$does not exist in general since it possesses a zero eigenvalue, the inverse applied to $\operatorname{sech} T$ does exist since $(\operatorname{sech} T, \operatorname{sech} T \tanh T)=0$. It is not difficult to show that

$$
L_{+}^{-1} \operatorname{sech} T=1 / 2(T \operatorname{sech} T \tanh T-\operatorname{sech} T) .
$$

This is, of course, not unique, since one can always add to it a multiple of the homogeneous solution sech $T \tanh T$. Because $(W, \operatorname{sech} T \tanh T)=0$, this arbitrary multiple does not affect the final result, however, which is

$$
(W, T \operatorname{sech} T \tanh T-\operatorname{sech} T)=0 .
$$

This is then the constraint (11a) rewritten in terms of $W$. But Weinstein [25] also shows that, subject to (15),

$$
\left(W, L_{-} W\right) \geq C_{2}(W, W),
$$

where $C_{2}$ is a positive constant. In terms of the original variable $U_{1}$, the following inequality then holds

$$
\left(U_{1}, L_{+} L_{-} L_{+} U_{1}\right) \geq C_{2}\left(L_{+} U_{1}, L_{+} U_{1}\right)=C_{2}\left(U_{1}, L_{+}^{2} U_{1}\right) .
$$

The final step is to show that

$$
\left(U_{1}, L_{+}^{2} U_{1}\right) \geq C_{3}\left(U_{1}, L_{+} U_{1}\right)
$$

subject to (11), where $C_{3}$ is another positive constant. This last inequality arises from considering the following minimization problem:

$$
\text { minimize }\left(U_{1}, L_{+}^{2} U_{1}\right)
$$

subject to the constraints

$$
\begin{aligned}
\left(U_{1}, L_{+} U_{1}\right) & =1 \\
\left(U_{1}, \operatorname{sech} T\right) & =0 \\
\left(U_{1}, T \operatorname{sech} T\right) & =0 .
\end{aligned}
$$

Note that $\left(U_{1}, L_{+}^{2} U_{1}\right)$ is clearly non-negative. We define $\alpha=\min \left(U_{1}, L_{+}^{2} U_{1}\right)$, and it is only necessary to show that $\alpha>0$. As in [25], we will assume that $\alpha=0$ and show that this leads to a contradiction. (Technically speaking, a full proof requires showing that if $\alpha=0$ then the minimum must be attained by some function in a proper admissible class [25, 27]. For the sake of brevity, the discussion of this point will be omitted here.) 
The contradiction follows in a relatively straightforward manner by considering the Lagrange multiplier problem associated with the above constrained minimization, namely

$$
L_{+}^{2} U_{1}=\lambda L_{+} U_{1}+\beta \operatorname{sech} T+\gamma T \operatorname{sech} T
$$

plus the constraints (18). Taking the inner product of (19) with sech $T \tanh T$ (the zero mode of $L_{+}$) gives immediately $\gamma=0$. The equation can then be multiplied through once by the inverse of $L_{+}$, which gives

$$
L_{+} U_{1}=\lambda U_{1}-\frac{\beta}{2}(\operatorname{sech} T-T \operatorname{sech} T \tanh T)+\delta \operatorname{sech} T \tanh T,
$$

where $\delta$ at this step is arbitrary. Taking the inner product again with respect to sech $T \tanh T$, however, shows that $\delta=0$ since $\lambda=0$ if the minimum occurs at zero (i.e., $\alpha=0$ ).

Upon multiplying by the inverse of $L_{+}$once more, it is found that

$$
U_{1}=-\frac{\beta}{2} L_{+}^{-1}(\operatorname{sech} T-T \operatorname{sech} T \tanh T)+\omega \operatorname{sech} T \tanh T .
$$

As before, the inverse of $L_{+}$applied to sech $T-T \operatorname{sech} T \tanh T$ exists since the inner product $(\operatorname{sech} T \tanh T, \operatorname{sech} T-T \operatorname{sech} T \tanh T)=0$. The constraint (11b) applied to the above equation forces $\omega=0$, and further implies that $U_{1}$ must be an even function of $T$. The constraint (11a) then gives the condition

$$
\left(\operatorname{sech} T, U_{1}\right)=-\frac{\beta}{2}\left(\operatorname{sech} T, L_{+}^{-1}(\operatorname{sech} T-T \operatorname{sech} T \tanh T)\right)=0 .
$$

Since $L_{+}$is self-adjoint and $L_{+}(\operatorname{sech} T-T \operatorname{sech} T \tanh T)=\operatorname{sech} T$, the above condition can be written

$$
\left(\operatorname{sech} T, U_{1}\right)=-\frac{\beta}{2}\|\operatorname{sech} T-T \operatorname{sech} T \tanh T\|^{2}=0,
$$

which can only be satisfied when $\beta=0$. Thus, if one assumes that the minimizing solution occurs at $\lambda=0$, we have shown that $\beta, \gamma, \delta$, and $\omega$ must also all be zero, which implies that no solution exists. This contradiction implies that the minimum must occur at some positive value, i.e., $\alpha>0$, and therefore

$$
\left(U_{1}, L_{+}^{2} U_{1}\right) \geq C_{3}\left(U_{1}, L_{+} U_{1}\right)
$$

where $C_{3}$ is some positive constant.

The resulting differential inequality for $V$, using (12), (14), (16) and (20), is thus

$$
\frac{d V}{d \bar{\xi}} \leq-2 C_{2} C_{3} V
$$

Integrating this gives

$$
V(\bar{\xi}) \leq V(0) e^{-2 C_{2} C_{3} \bar{\xi}}
$$

and upon using (13),

$$
\left\|U_{1}\right\|^{2} \leq \frac{V(0)}{C_{1}} e^{-2 C_{2} C_{3} \bar{\xi}}
$$




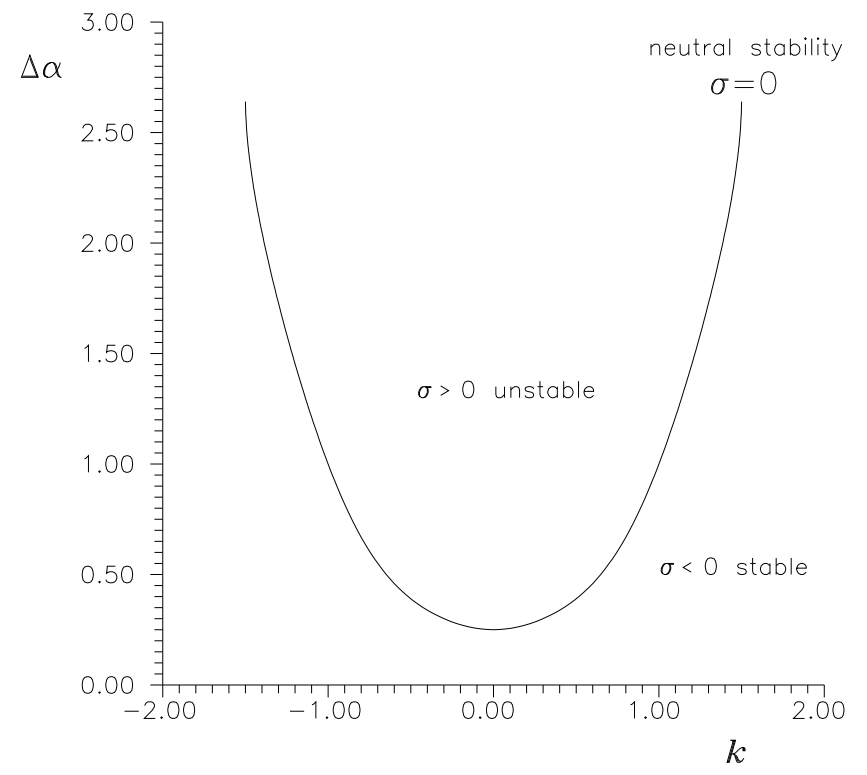

FIG. 2. Plot of the neutral stability curve $(\sigma=0)$ giving the critical value of the overamplification $\Delta \alpha$ as a function of the wavenumber $k$. Note that the most unstable wavenumber, $k=0$, corresponds to a value of $\Delta \alpha=1 / 4$.

Thus, $U_{1} \rightarrow 0$ exponentially as $\bar{\xi} \rightarrow \infty$ for initial conditions which do not lie in the space spanned by the two zero eigenfunctions.

The stability of the leading order hyperbolic secant solution is therefore entirely determined by the perturbed behavior of the two zero eigenvalues. Specifically, for small $\Gamma l$, the leading order hyperbolic secant solution is exponentially stable for $\Delta \alpha>(4 / 405)(\Gamma l)^{4} \equiv \Delta \alpha_{c}$. As mentioned previously, this asymptotic stability should be contrasted with the behavior of pulses when erbium-doped fiber amplifiers are employed $[5,6,7,8]$. In this case, the underlying Hamiltonian structure of the NLS equation is preserved even under the action of the periodic loss and gain, and as a result pulses approach their asymptotic guiding-center soliton solutions via the shedding of dispersive radiation. When phase-sensitive amplifiers are employed, however, this Hamiltonian structure is broken, which gives rise to significantly different asymptotic behavior.

4. Bifurcation from the trivial solution. In this section, Region B of Fig. 1 is considered by linearizing (2) about the basic state, i.e., by letting $U=\tilde{U}$ where $\tilde{U} \ll 1$. The linearized evolution about the basic state is

$$
\tilde{U}_{\bar{\xi}}+\frac{1}{4} \tilde{U}_{T T T T}-\frac{1}{2} \tilde{U}_{T T}+\left(\frac{1}{4}-\Delta \alpha\right) \tilde{U}=0,
$$

and looking for solutions of the form $U=\exp (\sigma \bar{\xi}+i k T)$ gives the dispersion relation

$$
\sigma+\frac{1}{4} k^{4}+\frac{1}{2} k^{2}+\left(\frac{1}{4}-\Delta \alpha\right)=0
$$




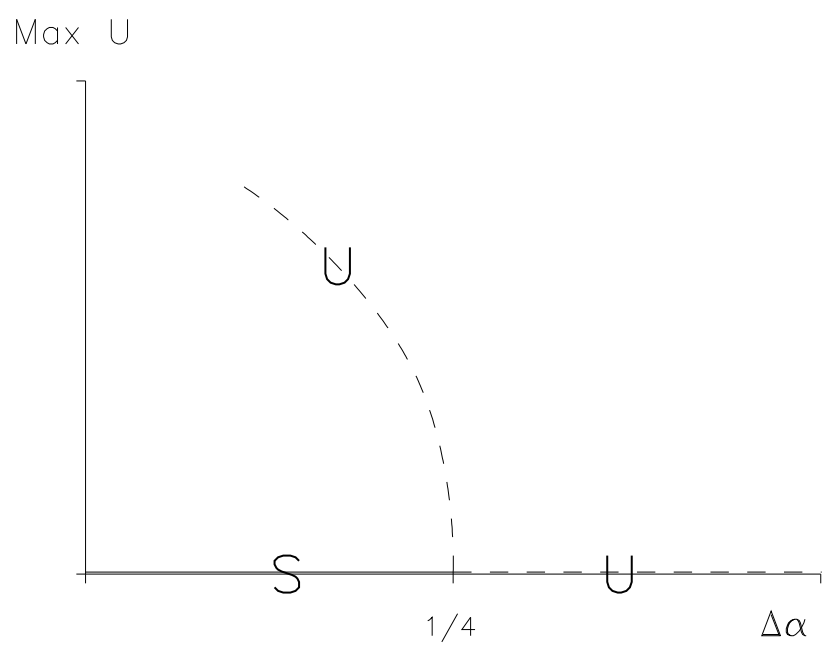

FIG. 3. Characteristic behaviour of the subcritical bifurcation emanating from $(U, \Delta \alpha)=$ $(0,1 / 4)$. Curves denoted by $S$ (and with solid curves) indicate stable solutions, while those denoted by $U$ (and with dashed curves) indicate unstable solutions.

For $\sigma<0(\sigma>0)$, the solution is stable (unstable). The value $\sigma=0$ corresponds to neutral stability and for a given $k$ occurs when

$$
\Delta \alpha=\frac{1}{4}\left(k^{2}+1\right)^{2}
$$

As can be seen from Fig. 2, $k=0$ is the most unstable wavenumber.

To determine the nonlinear behaviour of the solution near the minimum of the neutral stability curve, a multiple scale expansion about $U=0$ and $\Delta \alpha=1 / 4$ can be performed $[22,28,29]$. The appropriate slow space and time variables in this case are

$$
\begin{aligned}
& \eta=\delta^{2} \xi \\
& \tau=\delta T,
\end{aligned}
$$

where $\delta \ll 1$, while the appropriate form of the expansion is

$$
\begin{aligned}
U & =0+\delta V(\eta, \tau)+O\left(\delta^{2}\right)+\cdots, \\
\Delta \alpha & =\frac{1}{4}-\delta^{2} \mu+\cdots
\end{aligned}
$$

The evolution equation in this case is then [22, 28, 29]

$$
V_{\eta}=\frac{1}{2} V_{\tau \tau}+V^{3}-\mu V
$$

with $V \rightarrow 0$ as $\tau \rightarrow \pm \infty$. A steady-state solution of (27) is easily found for $\mu>0$, namely

$$
V \equiv V_{s}=\sqrt{2 \mu} \operatorname{sech} \sqrt{2 \mu} \tau .
$$

Stability of this steady-state is determined by linearizing,

$$
V=V_{s}(\tau)+W(\tau) e^{\lambda \eta},
$$




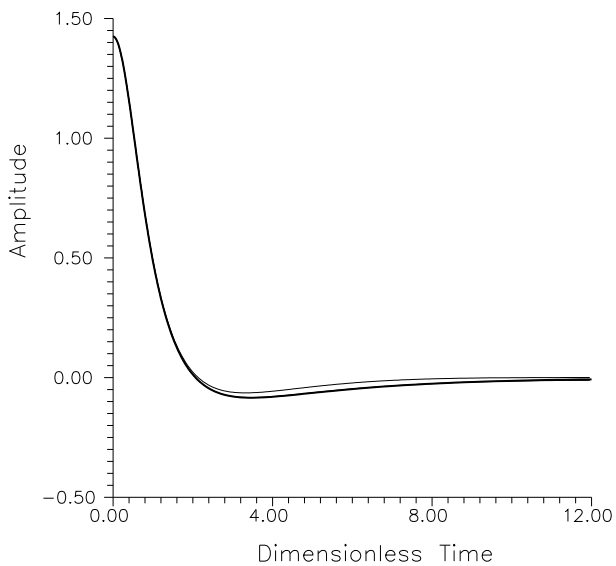

(a)

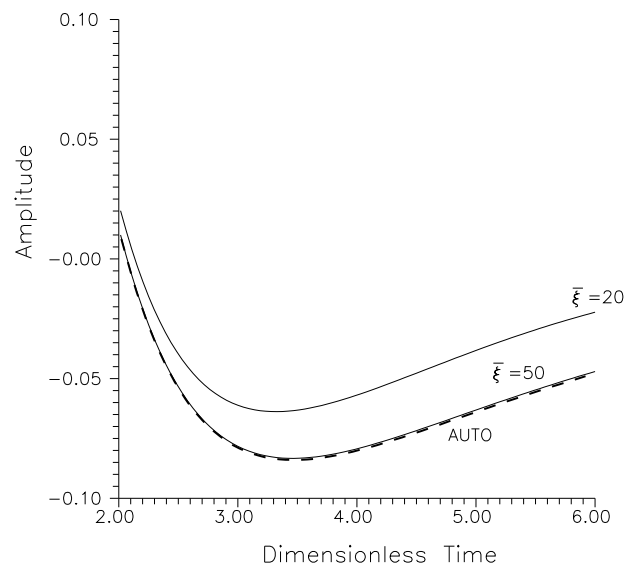

(b)

FIG. 4. Comparison of steady-states computed using AUTO with full numerical simulations of the averaged evolution. Three pulse profiles are depicted, which are the AUTO solution and the averaged evolution for values of $\bar{\xi}=20$ and $\bar{\xi}=50$. In (a) the interval $T \in[0,12]$ is shown, while (b) shows the interval $T \in[2,6]$, which is magnified to better depict the differences between the three solutions. Note that for large $\bar{\xi}$ the averaged solution approaches the steady-state solution generated via AUTO.

where $W \ll V_{s}$, which gives

$$
\frac{1}{2} \partial_{\tau}^{2} W+3 V_{s}^{2} W-\mu \tilde{V} \equiv L_{+}^{\mu} W=\lambda W .
$$

Since the operator $L_{+}^{\mu}$ has one positive eigenmode [25], the subcritical branch of steady-state solutions emerging from the bifurcation point $(U, \tilde{\alpha})=\left(0, \frac{1}{4}\right)$ is unstable, as shown in Fig. 3 .

5. Steady-states using AUTO. In this section, the bifurcation software package AUTO [30, 31] is used to determine the full set of steady-state solutions and associated bifurcation diagram for (2). AUTO allows for the investigation of parameter values which are beyond the range of the asymptotic and perturbation analysis of the previous sections. In particular, it will be shown that the solution branch associated with the subcritical bifurcation from $U=0$ at $\Delta \alpha=1 / 4$ connects with the saddle-node bifurcation point in Region A.

First, (2) with $\partial_{\bar{\xi}} U=0$ is rewritten as a system of four coupled, first order, ordinary differential equations with associated boundary conditions. This system is solved as a boundary value problem on the interval $0 \leq T \leq T_{0}$. Two of the four boundary conditions to be used exploit the even symmetry about the origin, i.e., $U_{T}=0$ and $U_{T T T}=0$ at $T=0$. The remaining two boundary conditions, which for computational reasons must be applied at a finite distance from $T=0$, i.e., at $T=T_{0}$, are derived from the solution behavior for small $U$ [32]. In particular, in the exponentially decaying tails far from the localized pulse, the steady-state equation reduces to

$$
U_{T T T T}-2 U_{T T}+(1-4 \Delta \alpha) U=0 .
$$

For $\Delta \alpha<1 / 4,(31)$ has two exponentially growing and two exponentially decaying modes. At the right-hand boundary $T=T_{0}$, the two decaying modes are the relevant ones. A linear combination of these two modes must describe the behavior of the 


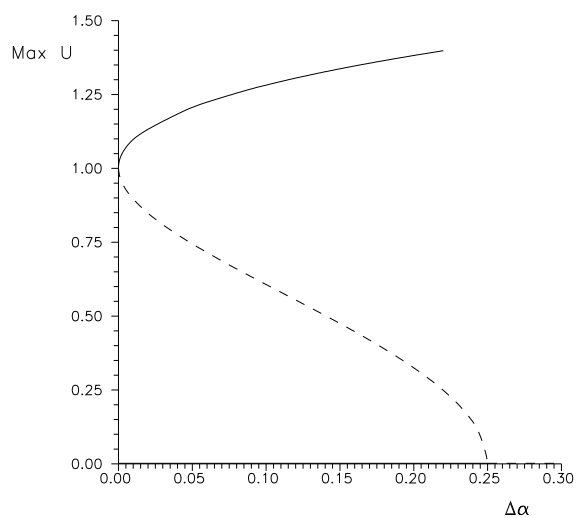

(a)

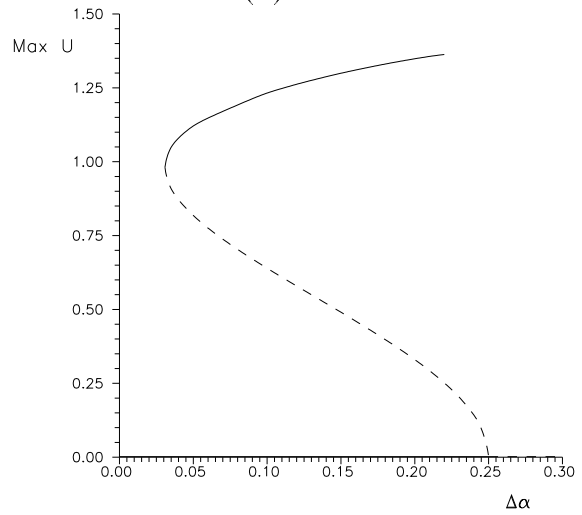

(c)

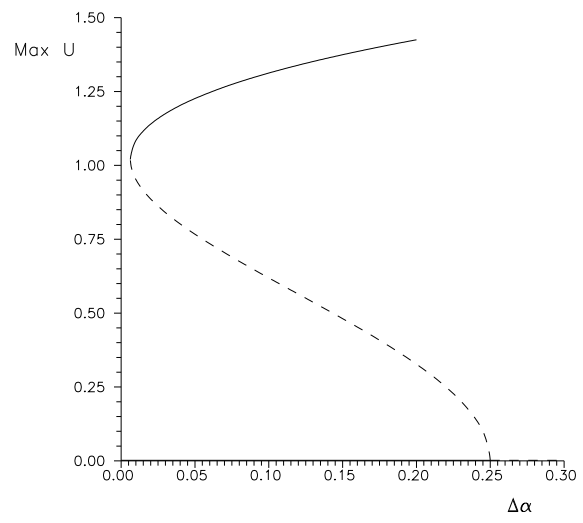

(b)

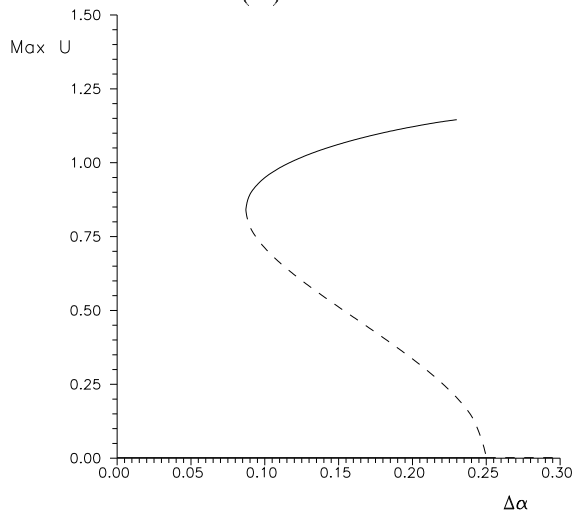

(d)

FIG. 5. Bifurcation diagrams associated with the averaged envelope equation. The solid curves correspond to stable solutions while the dashed curves correspond to unstable solutions. In each case, the $U=0$ solution is stable for $\Delta \alpha<1 / 4$ and unstable for $\Delta \alpha>1 / 4$. Here $(a),(b),(c)$ and (d) show the subcritical bifurcation from $\Delta \alpha=1 / 4$ and the location of the limit point for $\Delta \alpha=.02,1,2,100$, respectively. Note that as $\Gamma l$ is increased from near zero to infinity, the limit point moves from $\Delta \alpha \sim 0$ to $\Delta \alpha \sim 0.088$

pulse's exponentially decaying tails, where it is assumed that one is far enough away from the pulse so that the linear approximation is a good one. Appropriate numerical boundary conditions can be constructed in a number of ways, but the essential idea is to use a set of linear equations which force the solution behavior to contain no contribution from the two growing modes. A set of conditions which does this is to require

$$
\begin{array}{r}
U_{T T}+(\sqrt{1+\gamma}+\sqrt{1-\gamma}) U_{T}+\sqrt{1-\gamma^{2}} U=0 \\
U_{T T T}+(\sqrt{1+\gamma}+\sqrt{1-\gamma}) U_{T T}+\sqrt{1-\gamma^{2}} U_{T}=0
\end{array}
$$

at $T=T_{0}$. If one takes the general solution of (31) and substitutes it into (32a) and (32b) it is straightforward to verify that only the two exponentially decaying modes can remain.

It is also necessary for AUTO to have an exact solution from which to start the continuation procedure. Since no closed form exact solution of (2) is available, we use a continuation $[30,31]$ from another equation for which an exact closed form solution 


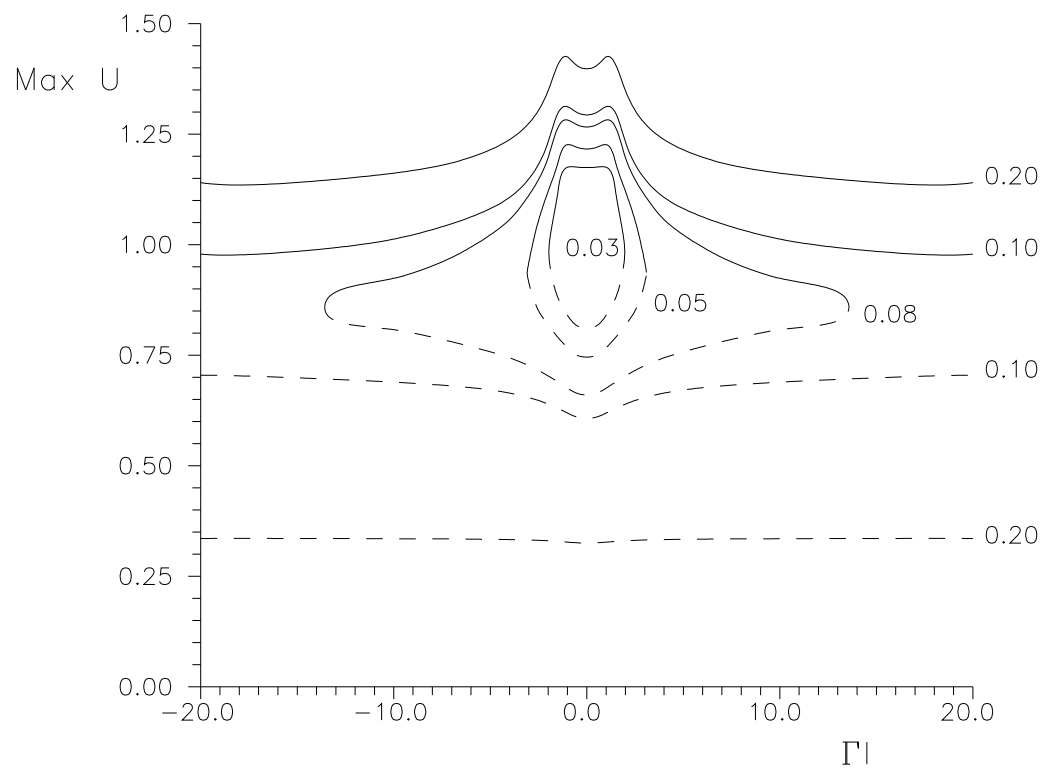

FIG. 6. Solution curves showing amplitude vs. $\Gamma$ l for different values of the overamplification parameter $\Delta \alpha$. The isola exists for values of $\Delta \alpha<0.088$. As the overamplification becomes larger, however, the curves separate into upper and lower branches corresponding to stable and unstable solutions.

is known, namely

$$
\frac{1}{4}\left(\partial_{T}^{2}-1\right)^{2} U-U-2 U^{3}+3 U^{5}+3 U U_{T}^{2}+3 U^{2} U_{T T}=0 .
$$

It is easy to verify that this equation possesses an exact hyperbolic secant solution and that this solution is stable.

Figs. $4 \mathrm{a}$ and $4 \mathrm{~b}$ show a comparison between the steady-state solutions computed with AUTO and the numerical solution of (2) for $\Gamma l=1$ and $\Delta \alpha=0.2$. Various propagation distances show the exponential approach of solutions of (2) to the steadystate solution computed with AUTO. In particular, Fig. 4a shows half the pulse profile computed using AUTO along with the averaged pulse envelope computed from (2) for the propagation distances of $\bar{\xi}=20$ and $\bar{\xi}=50$. Figure $4 \mathrm{~b}$ depicts the comparison for $T \in[2,6]$. Note that for large $\bar{\xi}$ the averaged evolution from (2) approaches the AUTO solution and they are in good agreement. Of course, AUTO provides a direct method for finding the final steady-state without having to propagate the solution of the averaged evolution to large distances. Similar numerical results hold for other values of the parameters $\Gamma l$ and $\Delta \alpha$.

Returning now to the aim of this section, which is the investigation of the bifurcation diagram associated with (2), the stable and unstable solution branches are computed for various values of $\Gamma l$ and $\Delta \alpha$. It is convenient to first consider $\Gamma l$ to be fixed and to determine the maximum amplitude (i.e., the steady-state value of $U$ at $\bar{\xi}=0)$ as a function of the overamplification $\Delta \alpha$. Figs. 5a-d show the effect of the perturbation upon the subcritical bifurcation from $\Delta \alpha=1 / 4$ and the saddle-node bifurcation point for various values of $\Gamma l$. These are in agreement with the local analyses of the previous two sections. As the value of $\Gamma l$ increases, the limit point moves away from $\Delta \alpha=0$. The bifurcation diagram associated with $\Gamma l$ as it approaches 


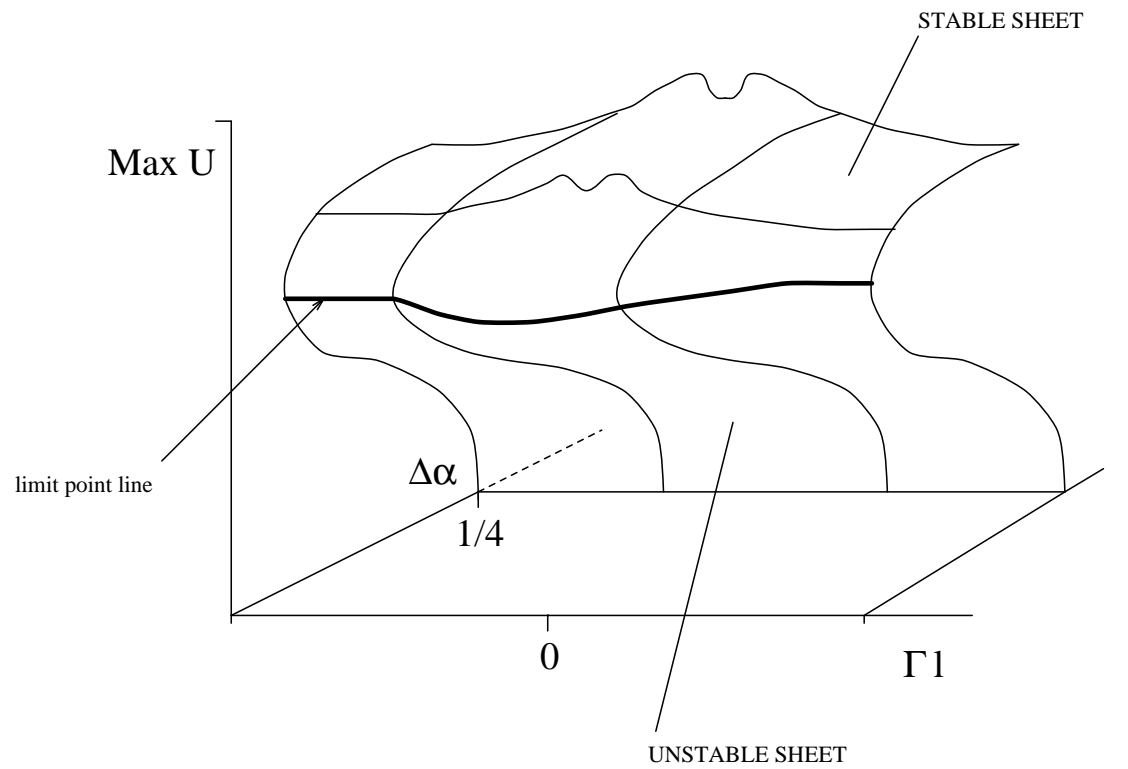

FIG. 7. Solution surface in parameter space for both the unstable and stable solutions of the averaged evolution. The limit point line denotes the set of all saddle-node bifurcation points

infinity is essentially given by Fig. 5d.

One can also fix $\Delta \alpha$ and determine the maximum amplitude as a function of the parameter $\Gamma l$. Fig. 6 depicts both the stable and unstable branches as $\Gamma l$ is increased to a large value. For $\Delta \alpha$ above a critical value, the stable and unstable solution branches remain disconnected for all values of $\Gamma l$. However, once the overamplification drops below a certain value, $(\Delta \alpha<.088)$, the stable and unstable branches are joined and limit points, or saddle-node bifurcations, exist in the amplitude vs. $\Gamma l$ plane. This can be thought of as an isola in parameter space where the size of the isola is dependent on the value of $\Gamma l$, i.e., as values of $\Gamma l$ get smaller, so does the isola. This behaviour is represented in Fig. 6 for various values of the overamplification $\Delta \alpha$. Note that although the amplitude of $U$ in (2) remains $O(1)$ as $\Gamma l$ approaches large values, the physical pulse envelope $\operatorname{Re}\left(q e^{-i \phi / 2}\right)$ grows without bound as $\Gamma l$ approaches infinity, as shown by (3).

Figure 7 combines the above parameter space projections to show the qualitative and complete bifurcation surface relating the maximum amplitude, $\Gamma l$ and $\Delta \alpha$. Note that although negative values of $\Gamma l$ are not permitted physically, they are convenient here since the surface is symmetric about $\Gamma l=0$. In addition, it should be noted that the results of the calculations using AUTO are valid only for $\Delta \alpha<1 / 4$, since beyond this point the boundary conditions (32) are no longer valid. Numerical simulations of (2) in this parameter range suggest that an interesting set of dynamics exists for $\Delta \alpha>1 / 4$. This parameter regime is not considered here, however.

6. Summary and Discussion. In conclusion, the use of phase-sensitive amplifiers in a nonlinear optical communications system has been considered. In this situation, the pulse evolution is governed by a nonlinear, fourth-order, dissipative equation with a characteristic length scale that is much longer than the typical dispersion length. We have examined the steady-state pulse solutions of this equation and determined their stability. Using an 'energy' type argument, the averaged pulse 
evolution was shown to be exponentially stable in the regime of small $\mathrm{\Gamma l}$. An exploration of the parameter space and bifurcation structure of the averaged evolution has also been carried out using AUTo. These results were shown to be in excellent agreement with the perturbation analysis of the averaged evolution equation. All evidence suggests that the steady-state hyperbolic secant solutions act as exponential attractors for a wide range of initial input conditions without shedding of a background dispersive radiation field.

From an optical perspective, PSAs give rise to many advantages over their erbiumdoped counterparts. First, PSAs are free of spontaneous emission noise [16]. Therefore, the Gordon-Haus timing jitter associated with a system employing PSAs will be substantially reduced, which allows for a potentially significant increase in the signal bit-rate. Second, initial amplitude and width variations are shown to be exponentially attenuated as the pulse propagates. This is in contrast to the case of erbium-doped amplifiers, where such initial variations are shed via a background dispersive radiation field.

Acknowledgements. This work was supported in part by the National Science Foundation (Applied Mathematics, 92-08415), the U. S. Air Force Office of Scientific Research (Mathematical Sciences, 93-1-0084) and J. Nathan Kutz was supported by a National Defense Science and Engineering Graduate Fellowship.

\section{REFERENCES}

[1] A. C. Newell and J. V. Moloney, Nonlinear Optics, Addison-Wesley, Redwood City, California, 1992.

[2] G. P. Agrawal, Nonlinear Fiber Optics, Academic Press, New York, 1989.

[3] A. Hasegawa, Optical Solitons in Fibers, 2nd Ed., Springer, Berlin, 1990.

[4] N. S. Bergano, J. Aspell, C. R. Davidson, P. R. Trischitta, B. M. Nyman, and F. W. KERFOOT, Bit error-rate measurements of $14,000 \mathrm{~km} 5 \mathrm{~Gb} / \mathrm{sec}$ fiber-amplifier transmission system using circulating loop, Electron. Lett., 27 (1991), pp. 1889-1890.

[5] A. Hasegawa and Y. Kodama, Guiding-center soliton in optical fibers, Opt. Lett., 15 (1990), pp. $1443-1445$.

[6] - Guiding-center soliton, Phys. Rev. Lett., 66 (1991), pp. 161-164.

[7] Y. Kodama and A. Hasegawa, Theoretical foundations of optical-soliton concept in fibers, in Progress in Optics XXX, E. Wolf, ed., Elsevier, Amsterdam, 1992.

[8] L. F. Mollenauer, S. G. Evangelides and H. A. Haus, Long-distance soliton propagation using lumped amplifiers and dispersion shifted fiber, J. Lightwave Tech., 9 (1991), pp. 194197.

[9] E. M. Dianov, A. V. Luchnikov, A. N. Pilipetskit, and A. N. Starodumov, Electrostriction mechanism of soliton interaction in optical fibers, Opt. Lett., 15 (1990), pp. 314-316.

[10] - Long-range interaction of solitons in ultra-long communication systems, Soviet Lightwave Comm., 1 (1991), pp. 235-246.

[11] J. P. Gordon AND H. A. Haus, Random walk of coherently amplified solitons in optical fiber transmission, Opt. Lett., 11 (1986), pp. 665-667.

[12] H. A. Haus, Quantum Noise in a solitonlike repeater system, J. Opt. Soc. Am. B, 8 (1991), pp. $1122-1126$.

[13] A. Mecozzi, J. D. Moores, H. A. Haus and Y. LaI, Soliton transmission control, Opt. Lett., 16 (1991), pp. 1841-1843.

[14] Y. Kodama and A. Hasegawa, Generation of asymptotically stable optical solitons and suppression of the Gordon-Haus effect, Opt. Lett., 17 (1992), pp. 31-33.

[15] L. F. Mollenauer, J. P. Gordon and S. G. Evangelides, The sliding-frequency guiding filter: an improved form of soliton jitter control, Opt. Lett., 17 (1992), pp. 1575-1577.

[16] H. YUEN, Reduction of quantum fluctuation and suppression of the Gordon-Haus effect with phase-sensitive linear amplifiers, Opt. Lett., 17 (1992), pp. 73-75.

[17] Sueng-HeE LeE, Ultra-long distance optical communication using phase-sensitive linear amplifier chains, Ph.D. Thesis, Northwestern University, December 1992. 
[18] I.H. Deutsch And I. ABRam, Reduction of quantum noise in soliton propagation via phase sensitive amplification, J. Opt. Soc. Am. B., to appear.

[19] R. W. Boyd, Nonlinear Optics, Academic Press, New York, 1992.

[20] J. N. Kutz, W. L. Kath, R.-D. LI, AND P. Kumar, Long-distance pulse propagation in nonlinear optical fibers using periodically-spaced parametric amplifiers, Opt. Lett., 18 (1993), pp. 802-804.

[21] J. N. Kutz, C. V. Hile, W. L. Kath, R.-D. Li, and P. Kumar, Pulse propagation in nonlinear optical fiber-lines that employ phase-sensitive parametric amplifiers, J. Opt. Soc. Am. B, 11 (1994), pp. 2112-2123.

[22] M. C. Cross and P. C. Hohenberg, Pattern formation outside of equilibrium, Rev. Mod. Phys., 65 (1993), pp. 851-1112.

[23] C. M. Bender and S. A. Orszag, Advanced Mathematical Methods for Scientists and Engineers, McGraw-Hill, New York, 1978.

[24] J. Kevorkin and J. D. Cole, Perturbation Methods in Applied Mathematics, SpringerVerlag, New York, 1981.

[25] M. WeInstein, Modulational stability of ground states of nonlinear Schrödinger Equations, SIAM J. Math. Anal., 16 (1985), pp. 472-491.

[26] B. Friedman, Principles and Techniques of Applied Mathematics, John Wiley \& Sons, New York, 1956.

[27] J. N. Kutz, Pulse propagation in nonlinear optical fibers using phase-sensitive amplifiers, Ph.D. Thesis, Northwestern University, December 1994.

[28] L. A. SEgEL, Distant side-walls cause slow amplitude modulation of cellular convection, J. Fluid Mech., 38 (1969), pp. 203-224.

[29] A. C. Newell and J. A. Whitehead, Finite bandwidth, finite amplitude convection, J. Fluid Mech., 38 (1969), pp. 279-303.

[30] E. J. Doedel and J. P. Kernévez, AUTO: Software for continuation and bifurcation problems in ordinary differential equations, Applied Mathematics Report, California Institute of Technology, Los Angeles, CA, 1986.

[31] M. J. Friedman and E. Doedel, Numerical computation and continuation of invariant manifolds connecting fixed points, SIAM J. Numer. Anal., 28 (1991), pp. 789-808.

[32] M. Lentini and H. B. Keller, Boundary value problems on semi-infinite intervals and their numerical solution, SIAM J. Numer. Anal., 17 (1980), pp. 577-604. 